\title{
BMJ Open Protocol for a systematic review of barriers, facilitators and outcomes in primary healthcare services for women in Pakistan
}

\author{
Sara Rizvi Jafree, ${ }^{1}$ Qaisar Khalid Mahmood, ${ }^{2}$ Ain ul Momina, ${ }^{3}$ Florian Fischer (D) ,,5 \\ Jane Barlow ${ }^{6}$
}

To cite: Jafree SR,

Mahmood QK, Momina Aul, et al. Protocol for a systematic review of barriers, facilitators and outcomes in primary healthcare services for women in Pakistan. BMJ Open 2021;11:e043715. doi:10.1136/ bmjopen-2020-043715

- Prepublication history and additional materials for this paper is available online. To view these files, please visit the journal online (http://dx.doi. org/10.1136/bmjopen-2020043715).

Received 11 August 2020 Revised 04 March 2021 Accepted 11 March 2021

Check for updates

(C) Author(s) (or their employer(s)) 2021. Re-use permitted under CC BY-NC. No commercial re-use. See rights and permissions. Published by BMJ.

For numbered affiliations see end of article.

Correspondence to

Dr Florian Fischer;

florian.fischer1@charite.de

\section{ABSTRACT}

Introduction The lack of universal health coverage and high poverty rates among the majority of women in Pakistan makes it essential to understand the quality and effectiveness of primary healthcare services. The aim of this project is to systematically review the available literature for interventions for primary healthcare services for women in order to provide the basis for future healthcare policy. The primary objective is to identify the effectiveness of the intervention in terms of how successful it was in improving health of women; whereas the secondary aim is to identify barriers and facilitators for delivery of primary healthcare services.

Methods and analysis A systematic review using a narrative synthesis will be undertaken, including qualitative, quantitative and mixed methods studies from January to June 2021. Electronic databases will be used including PubMed, BMC, Medline, CINAHL and Cochrane Library. The search will be conducted in English and no date restrictions will be applied. A thematic synthesis method will be used for data synthesis involving three steps: (1) the identification, coding and initial theme generation for effectiveness of primary healthcare interventions in Pakistan for women, (2) identification and grouping of overarching themes, and related subthemes, to develop descriptive themes for barriers and facilitators for primary healthcare delivery, and (3) generation of general analytical themes in order to present recommendations in terms of improved health outcomes for women.

Ethics and dissemination Ethics approval for this study was obtained from the Institutional Review Board, Forman Christian College University. Results will be disseminated via publications in international peer-reviewed journals. In addition, conference proceedings will be used to inform the government, researchers, donors, non-governmental organisations and other stakeholders. This study will result in a systematic identification and synthesis of barriers and facilitators for women's health outcomes that will help inform future primary health policies.

PROSPERO registration number CRD42020203472.

\section{INTRODUCTION}

There are over 101 million women living in Pakistan, most of whom are affected by issues that prevent them from having out-of-pocket
Strengths and limitations of this study

- This is the first systematic review to identify the effectiveness of different health interventions for women's health outcomes at primary level in Pakistan.

- All study designs (ie, qualitative, quantitative and mixed methods studies) will be included to ensure that 'promising' interventions are included and publication bias is avoided

- This synthesis is important not just for Pakistan, but other low and middle-income countries facing similar challenges related to women's healthcare delivery at primary level.

- Due to expected heterogeneity of included studies it may be difficult to summarise data to represent all the barriers and facilitators related to health outcomes.

finances for health expenditure. A major obstacle is that 30 million women in the country are below the poverty line, ${ }^{1}$ and no women irrespective of income are covered at federal level for universal healthcare. ${ }^{2}$ Of the $10 \%$ of people in the country who are covered by private health insurance, only $1 \%$ are women. ${ }^{3}$ Furthermore, only $23 \%$ of women are working, a majority of whom are informal sector workers, who are thereby deprived of health protection from employers. ${ }^{4}$ As such, the majority of women in Pakistan are dependent on subsidised or free healthcare from the government which is provided at a primary care level within their own communities. $^{56}$

Recent health indicators for Pakistani women confirm unmet targets in terms of the Millennium Development Goals and highlight critical challenges in terms of women's health and well-being. For example, neonatal mortality is 55 per 1000 live births, and has remained stagnant over the last 20 years. 


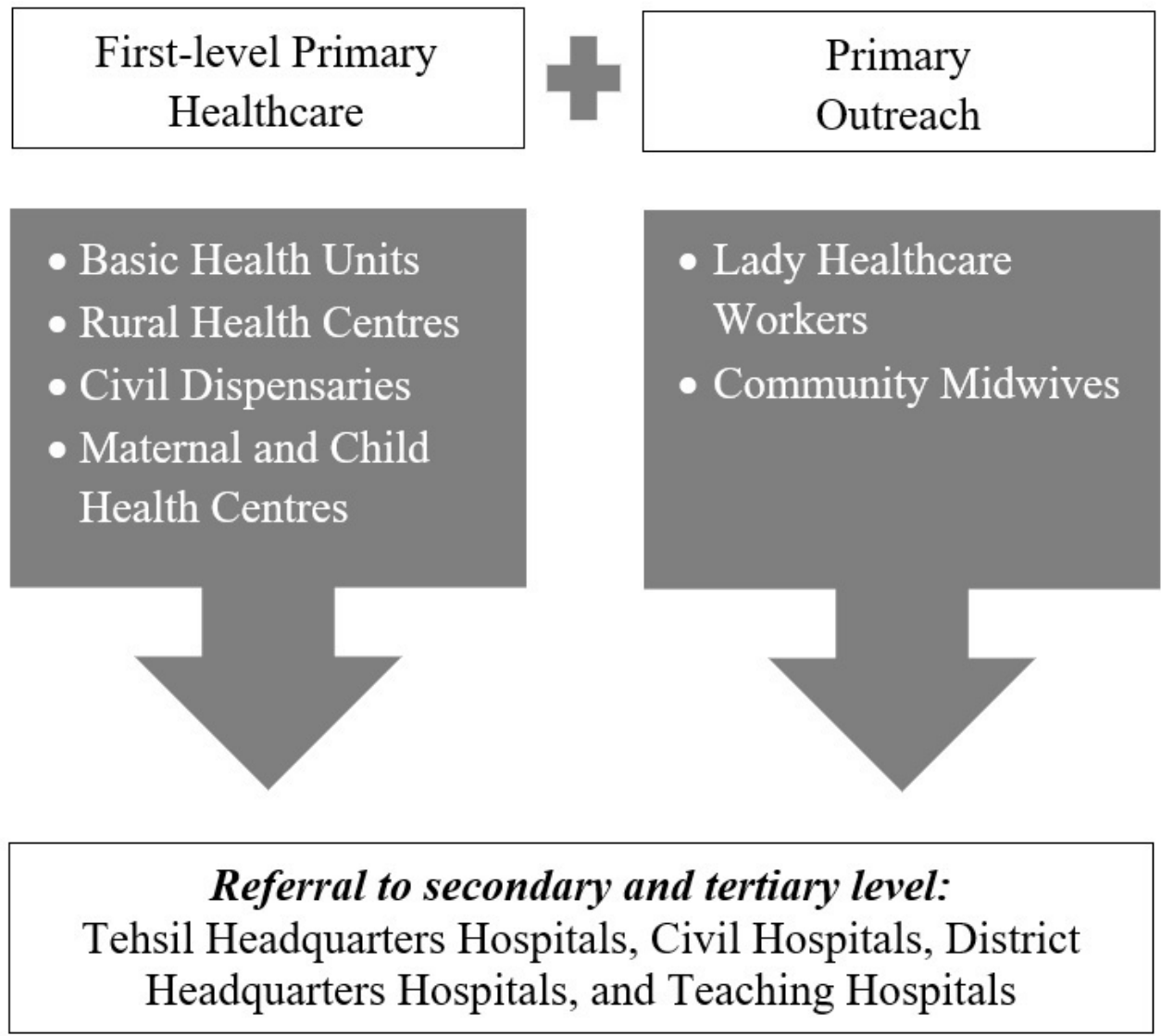

Figure 1 Primary healthcare structure in Pakistan.

Furthermore, the maternal mortality ratio is 274 per 1000 live births and has not declined significantly in the last few years. ${ }^{7}$ There is, however, an absence of data in terms of other key indicators of morbidity for women in Pakistan, ${ }^{8}$ including mental health, ${ }^{9}$ special needs and disability, ${ }^{10}$ chronic disease burden and multimorbidity, ${ }^{11}$ and violence and injury. ${ }^{12}$ There is also a lack of information with regard to preventive healthcare, ${ }^{13}$ or the quality of primary healthcare services. ${ }^{14}$

\section{Primary healthcare in Pakistan}

In 2011, the 18th Amendment to the Constitution devolved the health sector to the provinces of Pakistan (There are four provinces in Pakistan: Baluchistan, Khyber Pakhtunkhwa, Sindh and Punjab. The federal government allocates funds to the federal administration regions (Islamabad, Azad Jammu and Kashmir, Gilgit-Baltistan, and the Federally Administered Tribal Areas)). At federal level, the Ministry of National Health Service Coordination and Regulation remains responsible for monitoring and budget allocation, but has primarily adopted a singleline budgetary transfer to the provinces from the pool of tax revenues. Each province is responsible for managing primary healthcare services and their allocated budget, although the overall framework and structure is the same across the country (figure 1). Services provided to women at primary level include: reproductive and maternal health, vaccination and immunisation, health awareness, referral to secondary and tertiary sectors and routine counselling. Primary health services provided are the basic health units (BHUs) and the rural health centres (RHCs). The primary-level outreach is delivered through lady health workers (LHWs), who are responsible for 20 core tasks (maternal health services, referrals, documentation and record keeping, nutritional awareness, health education, hygiene communication, HIV/ AIDS and sexually transmitted diseases control, liaison and reporting with supervisors, and provision of medicine and supplies by government to catchment area) and five additional tasks (immunisation, emergency relief, tuberculosis control, malaria control and innovations in programme at primary level). Furthermore, community midwives (CMWs) provide primary-level outreach exclusively for child delivery.

Table 1 summarises the number of BHUs, RHCs, LHWs and CMWs in the country and provides a comparison of the population ratios in Pakistan and the recommended ratios of the WHO. These data suggest that there are problems with respect to low: (1) LHW and CMW to population ratios, (2) BHU and RHC doctor to population ratios, and (3) bed to population ratios for RHCs.

The effectiveness of primary healthcare services

Evidence about the impact of the above primary healthcare services has been accumulating over the past two decades. For example, a number of studies including 
Table 1 Primary healthcare services in Pakistan

\begin{tabular}{|c|c|c|c|c|}
\hline & $\begin{array}{l}\text { Lady health } \\
\text { workers }\end{array}$ & $\begin{array}{l}\text { Community } \\
\text { midwives }\end{array}$ & $\begin{array}{l}\text { Basic health } \\
\text { units }\end{array}$ & $\begin{array}{l}\text { Rural health } \\
\text { centres }\end{array}$ \\
\hline$n^{25}$ & 110000 & 4700 & 4996 & 638 \\
\hline WHO recommendations for ratios ${ }^{53}$ & $4.45: 1000$ & $4.45: 1000$ & $1: 125000$ & $1: 125000$ \\
\hline Number of doctors at BHU/RHC ${ }^{54}$ & & & 1 & $4-6$ \\
\hline Number of beds at each $\mathrm{RHC}^{54}$ & & & & $10-20$ \\
\hline Bed to population ratio for $\mathrm{RHC}$ in Pakistan & & & & 1:1000-2000 \\
\hline WHO recommendation for bed to population ${ }^{55}$ & & & & $5: 1000$ \\
\hline
\end{tabular}

$\mathrm{BHU}$, basic health unit; $\mathrm{RHC}$, rural health centre.

randomised controlled trials (RCTs) have found that the LHW programme is successful at a national level in improving contraception use, ${ }^{15}$ that educational sessions by LHWs on perinatal care have resulted in improved stillbirth and neonatal mortality rates, ${ }^{16}$ that traditional birth attendants (TBAs) delivered improved antenatal care to women and used disposable delivery kits resulting in reduced perinatal mortality ${ }^{17}$ and that a cognitivebehavioural therapy-based intervention increased the likelihood of contraception use and increased time reported for playing with infants. ${ }^{18}$

The evidence, however, is mixed with some studies showing ineffective services and poor health outcomes for women. ${ }^{19}$ For example, despite higher literacy and awareness in urban areas such as Lahore, only $7 \%$ of women were found to have completed tetanus coverage after LHW visitations. ${ }^{20}$ Similarly, interventions for acute malnutrition, promotion of breast feeding and community advocacy have also been found to be ineffective at a national level. ${ }^{21}$

Reasons for the variation in conclusions about the effectiveness of primary health interventions include differences in research design, lack of longitudinal follow-up and variation in the measurement of outcomes in terms of location (ie, cities or provinces rather than at national level). ${ }^{132223}$ Furthermore, many studies measure effectiveness in terms of the satisfaction of the participants, as opposed to the impact of service delivery in the community. ${ }^{24}$

Other issues with regard to primary healthcare include low uptake (eg, only $33 \%$ of pregnant women make four or more visits for antenatal care, and almost half of births take place without skilled birth attendance $)^{25}$ and high dropout (eg, one-third of women who do seek antenatal care from BHUs and RHCs stop before the follow-up visits). ${ }^{26}$

\section{Barriers and facilitators to effective service delivery}

Although the government of Pakistan had hoped to cover 184 million people or $83.6 \%$ of the national population through primary healthcare centres, ${ }^{27}$ the reality is that less than $5 \%$ of people access BHUs and RHCs. ${ }^{28} \mathrm{~A}$ number of reasons for this have been identified including poor quality of services, ${ }^{26}$ geographical inaccessibility for women living in remote areas ${ }^{29}$ and preference for alternatives such as home remedies, private centres, quack services, religious and faith healers, homeopathy and herbal solutions. ${ }^{13} 2230$ In addition, women are unable to obtain services from primary health centres for health needs such as injuries and violence, ${ }^{31}$ and there is a tendency for women not to be referred to these centres, and a lack of continuity of care. ${ }^{32}$

Research also indicates that LHWs and CMWs face considerable work-related and service delivery challenges, such as excessive workloads, job insecurity and nonpayment of salaries, ${ }^{33}$ in addition to suboptimal health facilities and shortage of supplies. ${ }^{34}$ There are also problems in terms of community rejection and high rates of violence against LHWs and CMWs. ${ }^{35}$ Further research suggests that recruitment of unsuitable candidates, poor training of LHWs and CMWs and a lack of community trust in LHW/CMW skills prevent uptake of services by rural women, ${ }^{37}$ who prefer to use TBAs because they are cheaper and a preferred choice by family, husbands and in-laws. ${ }^{38}$ To satisfy cultural preferences, interventions at the primary care level have tended to focus on training and supporting TBAs and local midwives, thereby neglecting investment in training and supervision for LHWs and CMWs. $^{39}$

Research also shows that $18 \%-20 \%$ of BHUs have problems related to the high rates of absenteeism of doctors, LHWs, CMWs and other healthcare staff, ${ }^{28} 39$ some of which is due to poor monitoring mechanisms by supervising authorities and insecure locations. ${ }^{38} 40$ Other issues related to ineffective state management include low total government health expenditure as a proportion of gross domestic product at $0.8 \%,{ }^{41}$ low allocation of funds to the primary healthcare sector, ${ }^{42}$ mismanagement of funds ${ }^{28}$ and the failure of contracting 
management to the private sector to improve service utilisation by women. ${ }^{434}$

\section{Need for this review}

To date, there is no health policy planning for the protection of women in Pakistan. ${ }^{45}$ Neither is there any recognition that without specific health policy planning for women at the primary healthcare level, health behaviour and health outcomes for women in the country will remain poor. Furthermore, research suggests that unless future policy development is based on evidence about what works, women's health will not be improved. ${ }^{16}$ Despite an increasing number of studies evaluating the effectiveness of primary healthcare services, there is currently no synthesis of this research in terms of its effectiveness in improving health outcomes for women. For this study, we define effectiveness as the degree to which the treatment was successful, ${ }^{46}$ or in our study's case the degree to which the intervention was successful in improving health outcomes in women. There has been no attempt to date to identify the key facilitators and barriers to improved outcomes in terms of the difficulties faced by LHWs and CMWs in delivering healthcare. This evidence gap will be addressed through the conduct of a systematic review synthesising the available evidence, in order to provide the basis for future healthcare policy.

\section{Aims and research questions}

The aim of this project is to systematically review the available literature related to interventions for primary healthcare services in Pakistan for women, in order to provide recommendations for future healthcare policy. The primary objective is to identify the effectiveness of the intervention in terms of how successful it was in improving the health of women; whereas the secondary aim is to identify barriers and facilitators for delivery of primary healthcare services. The specific research questions include:

- Assessment of what is known regarding the effectiveness of the intervention delivered in Pakistan to improve health of women at the primary level.

- Identification of the barriers and facilitators in terms of the delivery of primary healthcare services in Pakistan for women by LHWs and CMWs.

- Making recommendations in terms of what works to improve primary health outcomes for women in Pakistan.

\section{METHODS}

\section{Study design}

A systematic review using a narrative synthesis ${ }^{47}$ will be undertaken to identify and synthesise empirical research to address the above research questions. The reason for implementing a narrative synthesis is the dearth of intervention-based studies, inadequate methodological reporting of RCT interventions and the need thereby to identify promising interventions from a wider range of study designs. ${ }^{48} 49$ The review protocol has been prepared following guidelines for the Preferred Reporting Items for Systematic Review and Meta-Analysis Protocols. ${ }^{50}$ The systemic review is registered at PROSPERO.

\section{Inclusion and exclusion criteria}

The inclusion criteria have been established using the PICOS acronym as follows:

Population: The population of interest is all women in Pakistan. Studies that focus exclusively on children or other populations will be excluded from the review.

Intervention: Healthcare services/interventions that are delivered in the primary healthcare sector. Studies focusing on the secondary and tertiary health sectors will be excluded from the review.

Comparator: No intervention for improvement in health services, although the review will also include studies that have used one-group designs in which no comparator is used, in order to identify potentially promising interventions in need of further evaluation.

Outcome: All physical or mental health outcomes, in addition to barriers and facilitators of service delivery at primary healthcare level.

Study design: All study designs (ie, including qualitative, quantitative and mixed methods studies) will be included to ensure that 'promising' interventions that have not yet been evaluated using RCTs are identified.

The language will be restricted to English.

\section{Search methods}

Systematic searches will be conducted using the following platforms and electronic databases: PubMed, BMC, Medline, CINAHL and Cochrane Library. No date restrictions will be applied. We plan to conduct the search in the months of January to June 2021. We will undertake forward and backward reference searching of included studies by searching for original articles cited in studies found and examining references cited in articles. We will also adopt forward and backward author searching to identify an expert's later and previous work in the area.

A search of the grey literature will also be undertaken using Google Scholar to identify published reports related to the primary healthcare sector, such as WHO, United Nations and Government of Pakistan reports. Finally, we will use contacts with experts in the field and the government health ministry to access unpublished reports related to the primary healthcare sector. A record of the search will be kept and published as online supplemental file 1 .

Search terms have been identified through an initial review of the literature and with the help of Medical Subject Headings. The search terms are presented in online supplemental appendix 1 and will be modified for use in different databases.

\section{Data extraction and management}

EndNote software will be used to collate the references and remove duplicates. Assessment of the titles and abstracts will be undertaken by two review authors 
independently to determine if the full text meets the inclusion criteria. In case of discrepancy, consensus will be reached by consulting a third reviewer. For each study, we will extract the following information:

- Study/intervention design

- Setting

- Aim of study

- Description of participants

- Sample size

- Methodology

- Outcome of intervention/findings of study/effectiveness of intervention

- Limitations/barriers/facilitators

- Implications for improved interventions and policy for women's primary health services

\section{Missing data}

If there are missing data, we will attempt to contact the study authors. If there is no response after two attempts, a decision about whether the missing data are sufficiently significant to exclude the study from the review will be made.

\section{Assessing risk of bias}

As the quality of the included studies is likely to be mixed in terms of research design and methods, critical appraisal will be undertaken using the Mixed Methods Appraisal Tool (MMAT) version 2018, which has been explicitly designed for appraisal of the quality of empirical studies based on primary data collection. ${ }^{51}$ The tool will allow us to appraise the methodological quality of five different types of studies: (1) qualitative, (2) RCTs, (3) nonrandomised studies, (4) quantitative descriptive studies, and (5) mixed methods studies. Assessment of the risk of bias will be undertaken by SRJ and QKM independently, considering the items of MMAT. Two screening questions will determine if there are clear research questions in the study and if the collected data address the research questions. Depending on the type of study, five questions related to the methodological quality will be addressed with three rating options of 'Yes', 'No' or 'Can't tell'. We will use the risk of bias assessment results to assess the confidence of results and limitations of studies. Studies with serious methodological concerns will be excluded. We will not assess the quality of government or international reports.

\section{Data synthesis}

A template will be used to organise the extracted data. Studies will be grouped and the results of the review will be tabulated separately according to study type. A thematic synthesis method will be used, ${ }^{49} 52$ which is a transparent and systematic means of synthesising qualitative, quantitative and mixed methods studies in narrative form involving three steps. First, we will identify interventions that are targeting primary healthcare services for women. The broad outcomes and conclusions, according to intervention or programme, will be coded and themes will be developed. Initially, coding will be done based on study type, targeted health outcomes for women and design of intervention. Second, based on initial coding, we will identify overarching themes and related subthemes. Similar themes will be grouped together to develop descriptive information for barriers and facilitators for primary health outcomes in women. Third, general analytical themes will be generated in order to present and summarise effective recommendations for improved health outcomes for women in Pakistan.

The analyst team is multiprofessional, including sociologists, public health experts and medical doctors; and thus the risk of bias is low. The preliminary thematic synthesis will be undertaken independently by two of the authors (SRJ and QKM) for each study. In case of discrepancy, final agreement will be sought by consulting a third reviewer (AM) to minimise bias. All authors will discuss and agree on the extracted data and themes. To further minimise bias, we will discuss our findings with health experts in the field and key stakeholders, including women medical officers for BHU, primary healthcare department and lady health supervisors. Findings will be presented in tabular form and separated according to study design-qualitative, quantitative and mixed methods. The results will be reported descriptively presenting complete information about study population, study design and setting, intervention type, barriers and facilitators, and effectiveness for women's health outcomes.

\section{Patient and public involvement}

No patients nor members of the public are involved in conducting the review.

\section{Ethics and dissemination}

Ethics approval is not required, as our review will only include published data. The findings of this review will be published for the scientific community via articles in international peer-reviewed journals. In addition, the findings will be discussed and communicated via a range of state and stakeholder platforms and conferences nationally, in order to mobilise improvements for primary healthcare and health policy development for women in the country.

\section{DISCUSSION}

This will be one of the first systematic reviews to synthesise evidence regarding potentially effective primary healthcare services or interventions for women in Pakistan, in addition to what is known about the main barriers and facilitators to service use. Therefore, this systematic review is going to provide valuable insights for implementation science and health services research. Furthermore, it has a very practical relevance, because the systematic identification and synthesis of what is currently known about the effectiveness of different health interventions, its implementation and the challenges faced by LHWs and CMWs in service delivery at primary level will help inform future policies for the government, researchers, 
donors, non-governmental organisations and other stakeholders. The evidence mapping will also show where further research is needed.

\section{Author affiliations}

${ }^{1}$ Department of Sociology, Forman Christian College, Lahore, Pakistan

${ }^{2}$ Department of Sociology, International Islamic University Islamabad, Islamabad,

Pakistan

${ }^{3}$ Institute of Public Health, King Edward Medical University, Lahore, Pakistan

${ }^{4}$ Institute of Public Health, Charité-Universitätsmedizin Berlin, Berlin, Germany

${ }^{5}$ Institute of Gerontological Health Services and Nursing Resarch, University of

Applied Sciences Ravensburg-Weingarten, Weingarten, Germany

${ }^{6}$ Department of Social Policy and Intervention, University of Oxford, Oxford, UK

\section{Twitter Sara Rizvi Jafree @JafreeRizvi}

Acknowledgements We acknowledge the support from the German Research Foundation (DFG) and the Open Access Publication Fund of CharitéUniversitätsmedizin Berlin.

Contributors The systematic literature review was conceptualised by SRJ. The activities were supervised by FF and JB. SRJ, QKM, AuM and FF conducted the systematic search. SRJ drafted the protocol. FF and JB revised it critically for important intellectual content. All authors approved the final version of this manuscript.

Funding The authors have not declared a specific grant for this research from any funding agency in the public, commercial or not-for-profit sectors.

Competing interests None declared.

Patient consent for publication Not required.

Provenance and peer review Not commissioned; externally peer reviewed.

Supplemental material This content has been supplied by the author(s). It has not been vetted by BMJ Publishing Group Limited (BMJ) and may not have been peer-reviewed. Any opinions or recommendations discussed are solely those of the author(s) and are not endorsed by BMJ. BMJ disclaims all liability and responsibility arising from any reliance placed on the content. Where the content includes any translated material, BMJ does not warrant the accuracy and reliability of the translations (including but not limited to local regulations, clinical guidelines, terminology, drug names and drug dosages), and is not responsible for any error and/or omissions arising from translation and adaptation or otherwise.

Open access This is an open access article distributed in accordance with the Creative Commons Attribution Non Commercial (CC BY-NC 4.0) license, which permits others to distribute, remix, adapt, build upon this work non-commercially, and license their derivative works on different terms, provided the original work is properly cited, appropriate credit is given, any changes made indicated, and the use is non-commercial. See: http://creativecommons.org/licenses/by-nc/4.0/.

\section{ORCID iD}

Florian Fischer http://orcid.org/0000-0002-4388-1245

\section{REFERENCES}

1 World Bank. Pakistan development update. growth: a shared responsibility. Washington: World Bank, 2017.

2 Malik MA. Universal health coverage assessment Pakistan. Karachi: Aga Khan University, 2015.

3 Pakistan Bureau of Statistics. Pakistan National health accounts 2013-14. Islamabad: Government of Pakistan, Pakistan Bureau of Statistics, 2015.

4 United Nations Pakistan. One United nations programme III 20182022, United nations sustainable development framework for Pakistan. Islamabad: United Nations Pakistan, 2018.

5 Nishtar S, Bhutta ZA, Jafar TH, et al. Health reform in Pakistan: a call to action. Lancet 2013;381:2291-7.

6 Syeda $\mathrm{MH}$. Making an impact analysis of social protection programs in Pakistan. J Res Soc Pakistan 2015;52:201-10.

7 National Institute of Population Studies. Health survey (PDHS) (201213). Islamabad: National Institute of Population Studies, 2017.

8 Ramalho A, Castro P, Gonçalves-Pinho M, et al. Primary health care quality indicators: an umbrella review. PLoS One 2019;14:e0220888.
9 Chisholm D, Sekar K, Kumar KK, et al. Integration of mental health care into primary care. Demonstration cost-outcome study in India and Pakistan. Br J Psychiatry 2000;176:581-8.

10 Mirza I, Tareen A, Davidson LL, et al. Community management of intellectual disabilities in Pakistan: a mixed methods study. J Intellect Disabil Res 2009;53:559-70.

11 Singh K, Patel SA, Biswas S, et al. Multimorbidity in South Asian adults: prevalence, risk factors and mortality. $J$ Public Health 2019;41:80-9.

12 Zakar R, Zakar MZ, Kraemer A. Primary health care physicians' response to the victims of spousal violence against women in Pakistan. Health Care Women Int 2011;32:811-32.

13 Ahmed J, Shaikh BT. The state of affairs at primary health care facilities in Pakistan: where is the State's stewardship? East Mediterr Health J 2011;17:619-23.

14 Shaikh BT, Kadir MM, Pappas G. Thirty years of Alma Ata pledges: is devolution in Pakistan an opportunity for rekindling primary health care? J Pak Med Assoc 2007;57:259.

15 Douthwaite M, Ward P. Increasing contraceptive use in rural Pakistan: an evaluation of the lady health worker programme. Health Policy Plan 2005;20:117-23.

16 Bhutta ZA, Memon ZA, Soofi S, et al. Implementing communitybased perinatal care: results from a pilot study in rural Pakistan. Bull World Health Organ 2008;86:452-9.

17 Jokhio $\mathrm{AH}$, Winter HR, Cheng KK. An intervention involving traditional birth attendants and perinatal and maternal mortality in Pakistan. N Engl J Med 2005;352:2091-9.

18 Rahman A, Malik A, Sikander S, et al. Cognitive behaviour therapybased intervention by community health workers for mothers with depression and their infants in rural Pakistan: a cluster-randomised controlled trial. Lancet 2008;372:902-9.

19 Shaikh BT, Hatcher J. Health seeking behaviour and health service utilization in Pakistan: challenging the policy makers. J Public Health 2005;27:49-54

20 Hasnain S, Sheikh NH. Causes of low tetanus toxoid vaccination coverage in pregnant women in Lahore district, Pakistan. East Mediterr Health J 2007;13:1142-52.

21 National Institute of Population Studies. Pakistan demographic and health survey. Islamabad: National Institute of Population Studies, 2013.

22 Callen M, Gulzar S, Hasanain A, et al. Improving public health delivery in Punjab, Pakistan: issues and opportunities. LJE 2013;18:249-69.

23 Mumtaz Z, Levay A, Bhatti A, et al. Good on paper: the gap between programme theory and real-world context in Pakistan's community midwife programme. BJOG 2015;122:249-58.

24 Budosan B. Mental health training of primary health care workers: case reports from Sri Lanka, Pakistan and Jordan. Intervention 2011:9:125-36.

25 World Health Organization. Primary health care systems (PRIMASYS): comprehensive case study from Pakistan. Geneva: World Health Organization, 2017.

26 Majrooh MA, Hasnain S, Akram J, et al. Coverage and quality of antenatal care provided at primary health care facilities in the 'Punjab' province of 'Pakistan'. PLoS One 2014;9:e113390.

27 Government of Pakistan. Pakistan statistical yearbook 2012 Islamabad: Pakistan Bureau of Statistics, 2012.

28 Malik MA, Gul W, lqbal SP, et al. Cost of primary health care in Pakistan. J Ayub Med Coll Abbottabad 2015;27:88.

29 Zaidi S, Riaz A, Rabbani F, et al. Can contracted out health facilities improve access, equity, and quality of maternal and newborn health services? Evidence from Pakistan. Health Res Policy Syst 2015;13:S54

30 Panezai S, Ahmad MM, Saqib SE. Factors affecting access to primary health care services in Pakistan: a gender-based analysis. Dev Pract 2017;27:813-27.

31 Rizvi N, Nishtar S. Pakistan's health policy: appropriateness and relevance to women's health needs. Health Policy 2008;88:269-81.

32 Oxford Policy Management. Lady health worker programme: external evaluation of the National programme for family planning and primary health care. Oxford Policy Management Oxford: Oxford, 2002.

33 Wazir MS, Shaikh BT, Ahmed A. National program for family planning and primary health care Pakistan: a SWOT analysis. Reprod Health 2013;10:60.

34 Hafeez A, Mohamud BK, Shiekh MR, et al. Lady health workers programme in Pakistan: challenges, achievements and the way forward. J Pak Med Assoc 2011;61:210.

35 Ahmed J, Ur Rehman S, Shahab M. Community midwives' acceptability in their communities: a qualitative study from two provinces of Pakistan. Midwifery 2017;47:53-9. 
36 Khan A. Lady health workers and social change in Pakistan. Economic Political Weekly 2011;46:28-31.

37 Nizar H, Chagani P. Analysis of health care delivery system in Pakistan and Singapore. Int J Nurs Educ 2016;8:21-6.

38 Mumtaz Z, Levay AV, Bhatti A. Successful community midwives in Pakistan: an asset-based approach. PLoS One 2015;10:e0135302.

39 Lakhani A, Jan R, Mubeen K. Strengthening the knowledge and skills of community midwives in Pakistan through clinical practice internships. J Asian Midwive 2016;3:26-38.

40 Salman A. Needs based budgeting in the health sector: issues and policy options. Islamabad: Centre for Peace and Development Initiatives, 2010.

41 Pakistan Bureau of Statistics. Pakistan National health accounts 2011-12. Islamabad: Pakistan Bureau of Statistics, 2012.

42 Ahmed J, Shaikh BT. An all time low budget for healthcare in Pakistan. J Coll Physicians Surg Pak 2008;18:388.

43 Loevinsohn B, Haq IU, Couffinhal A, et al. Contracting-in management to strengthen publicly financed primary health services--the experience of Punjab, Pakistan. Health Policy 2009;91:17-23.

44 Malik MA, Van de Poel E, Van Doorslaer E. Did contracting effect the use of primary health care units in Pakistan? Health Policy Plan 2017;32:1032-41.

45 Shaikh BT, Haran D, Hatcher J. Women's social position and health-seeking behaviors: is the health care system accessible and responsive in Pakistan? Health Care Women Int 2008;29:945-59.

46 Pascual Morena C, Martinez-Vizcaino V, Álvarez-Bueno C, et al. Effectiveness of pharmacological treatments in Duchenne muscular dystrophy: a protocol for a systematic review and meta-analysis. BMJ Open 2019;9:e029341.

47 Popay J, Roberts H, Sowden A. Guidance on the conduct of narrative synthesis in systematic reviews. A product from the ESRC methods programme. Computer Sci 2006.

48 Campbell M, McKenzie JE, Sowden A, et al. Synthesis without metaanalysis (SWiM) in systematic reviews: reporting guideline. $B M$ 2020;368:16890.

49 Snilstveit B, Oliver S, Vojtkova M. Narrative approaches to systematic review and synthesis of evidence for international development policy and practice. J Dev Effect 2012;4:409-29.

50 Moher D, Shamseer L, Clarke M, et al. Preferred reporting items for systematic review and meta-analysis protocols (PRISMA-P) 2015 statement. Syst Rev 2015;4:1.

51 Hong QN, Pluye P, bregues S F. Mixed methods appraisal tool (MMAT). Montreal: McGill University, 2018.

52 Thomas J, Harden A. Methods for the thematic synthesis of qualitative research in systematic reviews. BMC Med Res Methodol 2008;8:45.

53 World Health Organization. Health workforce requirements for universal health coverage and the sustainable development goals. human resources for health observer. 2016. Geneva: World Health Organization, 2016.

54 Government of Punjab. Minimum service delivery standards for primary and secondary health care in Punjab. Lahore: Government of Punjab, 2010.

55 Bajpai V. The challenges confronting public hospitals in India, their origins, and possible solutions. Adv Public Health 2014;2014:1-27. 